\title{
License Plate Localisation based on Morphological Operations
}

\author{
Xiaojun Zhai, Faycal Benssali and Soodamani Ramalingam \\ School of Engineering \& Technology \\ University of Hertfordshire, UH \\ Hatfield, UK
}

\begin{abstract}
Automatic Number Plate Recognition (ANPR) systems allow users to track, identify and monitor moving vehicles by automatically extracting their number plates. This paper presents an improved method to locate car plates in an ANPR system. The proposed method is based on morphological open and close operations where different Structuring Elements (SE) are used to maximally eliminate non-plate region and enhance plate region. This method has been tested using a database of UK number plates and results achieved have shown significant improvements in terms of the detection rate compare to other existing plate localisation systems.
\end{abstract}

Keywords - number plate localisation, ANPR, morphological operation.

\section{INTRODUCTION}

Automatic Number Plate Recognition (ANPR) systems allow users to track, identify and monitor moving vehicles by automatically extracting their number plates. These systems are rapidly becoming used for a vast number of applications. These include, e.g. automatic congestion charge systems, access control, tracing of stolen cars, or identification of dangerous drivers, and automatic Electronic Toll Collection (ETC) system [1][2]. Although some techniques, such as RFID (radio frequency identification) and WSN (wireless sensor network) are used for car identification, they are not cost effective as special equipments are required to be installed on the car.

The fundamental requirements of an ANPR system are image capture using an ANPR camera, and processing of the captured image. The image processing part, which is a computationally intensive task, includes two stages i.e. plate localisation and character recognition. Plate localisation normally requires two major tasks. The first one is to separate Number Plate (NP) area from Non-Number Plate (Non-NP) area and the second one is plate adjustment. The plate recognition stage requires a pre-processing step which is plate segmentation. In this step the symbols or characters will be separated from the NP so that only useful information are retained for recognition where the image format will be converted into symbols or characters by pre-defined transformation models.
This paper presents an improved method based on the open and close morphological operations to localise the position of number plates. The method only utilises two open and one close morphological operations to enhance plate region and eliminate non-plate region. Finally the plate region is acquired on the basis of known plate's geometrical relationship.

The rest of this paper is organised as follows. Related work is reviewed in Section II. Section III is concerned with the proposed morphologic method. The experimental results are provided and discussed in the section IV. Section V concludes the paper.

\section{RELATED WORKS}

Many plate detection, segmentation and recognition algorithms have been proposed to implement ANPR systems. NP detection algorithms can mainly be classified into three classes: edge-based algorithms, colour-based algorithms and texture-based algorithms. The implementation of those algorithms can be software or hardware-based.

The most commonly used method for license plate detection is certainly the combination of edge detection and mathematical morphology [3][4]. In this method, because the plate contains many vertical edge information, the higher edge density is looked for then according to the mathematical morphology of the plate, the position of the plate in the image will be detected. However, in this method, the detection rate is affected by the quality of the image itself (e.g. resolution and blurring) and the complexity of the scene (e.g. background and objects) which generates unwanted edge information [5][6]. To overcome the problems encountered in this method, colour-based image processing methods have been proposed [7][8]. However, these methods are not suitable for cases where the background has the same colour of the plate or in countries where multiple colours are used (e.g. China). Since the above methods are generally colour or shape-based, they are ineffective at detecting various license plates with varying colours and shapes. This is due to the nature of the plate which is texture-based. Researchers have exploited this by proposing texture-based feature extraction methods. The typical methods use SVM (Support Vector Machine) and Gabor Transformation [9][10][11]. Despite the good performance of these methods, the computation complexity will limit their usability [7]. 
Based on the above mentioned methods, many plate localisation algorithms have been developed. However, these algorithms are either having low performance or high computation complexity. In this paper, an improved algorithm with a high detection rate based purely on the mathematical morphology is proposed.

\section{MORPHOLOGICAL OPERATIONS BASED Plate LOCALISATION}

License plate is a pattern with high variations of contrast. This feature is used to locate the plate and is robust to the changes of lighting conditions and view orientations. The open and close morphological operations are used to extract the contrast features within the plate [4]. This is a relatively stable method when subjected to different image alterations or conditions.

The proposed algorithm in this paper consists of three major stages:

- Morphological operations for extracting plate features;

- Selection of candidate regions; and

- Verification of plate region.

Fig. 1 shows the block diagram of the overall proposed system. Each stage is explained further in the next sub-sections.

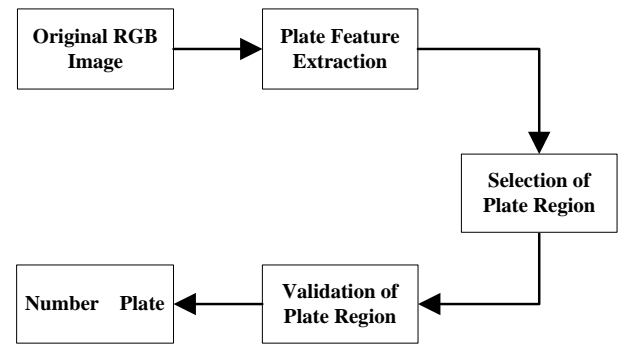

Figure 1. Block diagram of overall system.

\section{A. Plate Feature Extraction}

Fig. 4 illustrates the plate extraction stage. The original image is passed through two open and one close morphological operations, in order to maximise the elimination of non-plate regions.

The original RGB image is firstly loaded into the system, and then transformed to a Greyscale image which will be used as an input to the following block where the first morphological open operation will be used. This operation is an erosion followed by a dilation used to eliminate small and narrow parts of an image [12], In this step, the proposed method uses the morphological opening operation to smooth the background illumination, where a 'rectangular' structuring elements (SE) is used (See Fig. 2).

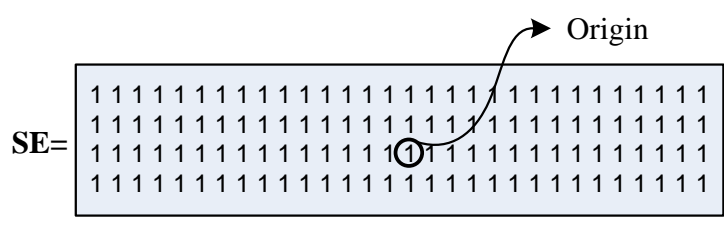

Figure 2. A 'rectangle' shaped SE with size $=4 \times 30$.

Where SE is a matrix fully filled by 1 's and its size is $4 \times 30$. The centre of the matrix is called 'Origin'. The choice of the size is purely based on the resolution of the original image and plate region (i.e. the resolution of entire image is $768 \times 288$, the maximum distance between the two neighbour characters are normally less than 30 pixels and the top and bottom margins are larger than 4 pixels, therefore, the best size of the first SE is be $4 \times 30$ ).

According to the rule of opening, this SE can effectively erase plate region and keep non-plate region from greyscale image. The result obtained after performing the opening operation is actually the background of the image. The next stage is to subtract the background image from the original greyscale image where the area of plate region will be highlighted. Fig. 3 illustrates the above stage.

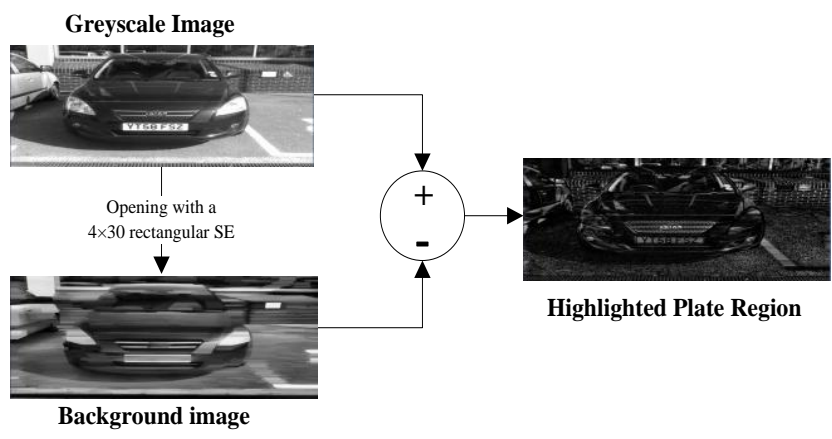

Figure 3. The highlighting plate region process.

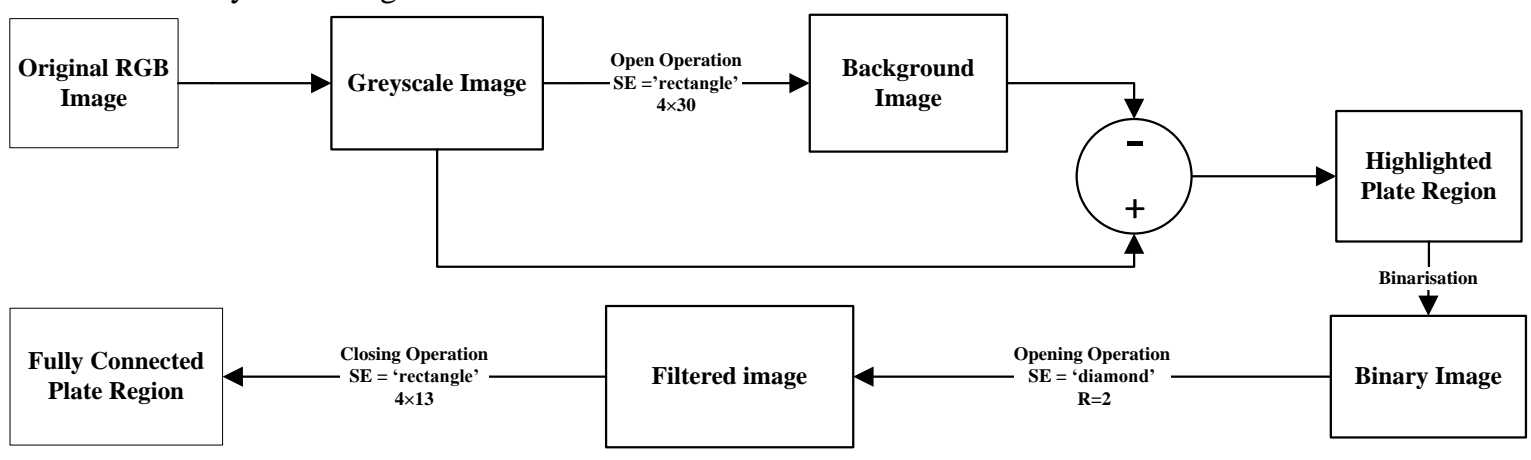

Figure 4. Block diagram of plate feature extraction. 
Highlighting the plate region is the main task in the plate extraction stage. Once it is performed the remaining tasks are straightforward. The highlighted plate region image needs to be binarised. This operation will further eliminate non-plate regions. A threshold level is calculated using the following formula (1):

$$
\text { Threshold }=(\max 1-(\max 1-\min 1) / 1.35) / 255
$$

Where:

- max1: is the maximum pixel value of the highlighted image;

- min1: is the minimum pixel value of the highlighted image.

Fig. 5 shows the result after binarisation

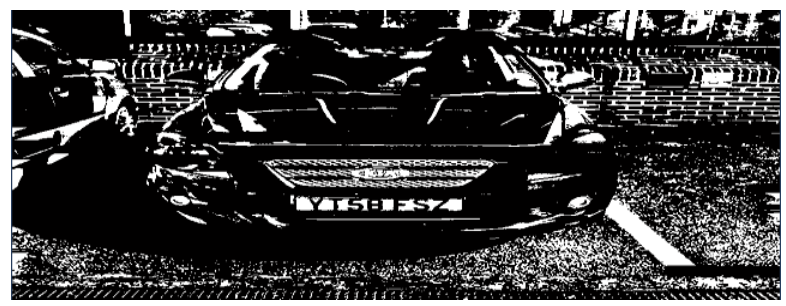

Figure 5. The binary image.

There are still unwanted information remained in the binary image. Therefore, the proposed system employs other two morphological operations to eliminate the rest of non-plate regions. The first one is another opening operation which is performed to remove small and narrow parts. A 'diamond' shaped SE is used for this purpose. Fig. 6 shows the SE and the resulting image.

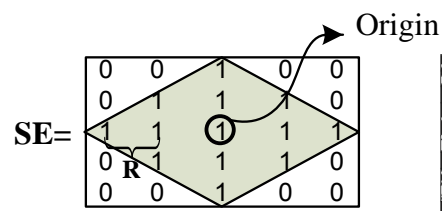

(a) A 'diamond' shaped $\mathrm{SE}$ with $\mathrm{R}=2$

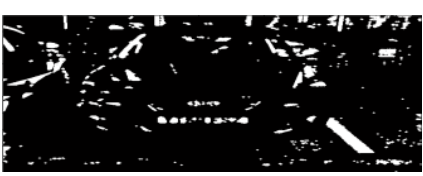

(b) The resulting image
Figure 6. The 'diamond' shaped SE and resulting image.

The SE is a 'diamond' shaped matrix that has a radius $\mathrm{R}=2$, where all 1's are inside the 'diamond' and all 0's outside it. When this special structure is used during the opening operation only diamond-shaped regions filled by 1 's will be kept. This operation is very useful to erase net-shaped and narrow lines that are surrounding the plate area.

Although the above operation can efficiently erase most of the unwanted information, some pixels in the plate region can also be eliminated. Therefore, the system needs an extra operation to fully fill the plate region to connect the pixels. On the contrast of opening operation, the morphological close operation is a dilation followed by erosion, which is normally used for fusing small holes and filling narrow aperture [2]. In this step, a $4 \times 13$ 'rectangle' shaped SE is used for the closing operation. The result obtained after performing the close operation is shown in Fig. 7. The choice of the size of the SE depends on the size of the plate region.

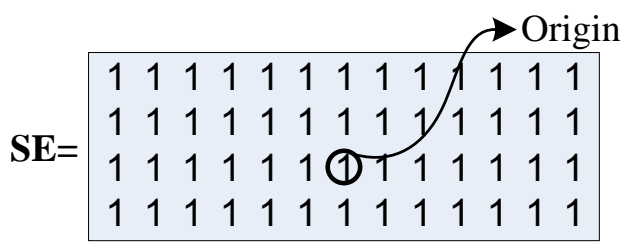

(a) A $4 \times 13$ 'rectangle' shaped SE

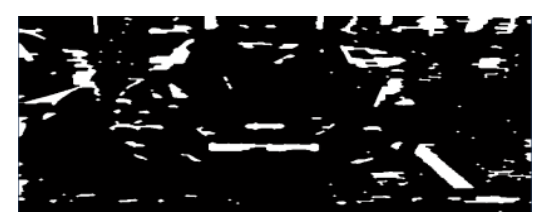

(b) The resulting image

Figure 7. The 'rectangle' shaped SE and its result.

As it can be seen from Fig. 7, the plate region can clearly be identified as it is a group of connected pixels which can be easily extracted using some known geometrical conditions (e.g. Width/Length ratio).

\section{B. Selection of Candidates Plate Region and Validation of Plate Region}

The output image from the previous stage consists of a set of groups of connected pixels. A labelling algorithm can be used to mark these pixels. In this proposed work, the labelling algorithm uses a '4-connectivity' method, and labels them using different numbers. Once all groups of pixels have been determined, each pixel is labelled based on the group it belongs to. Therefore, a set of potential candidates can be selected from the image using the known geometrical conditions. A validation process needs to be used to extract the candidate that represents the licence plate. Fig. 8 shows a block diagram that illustrates the selection and validation processes. 


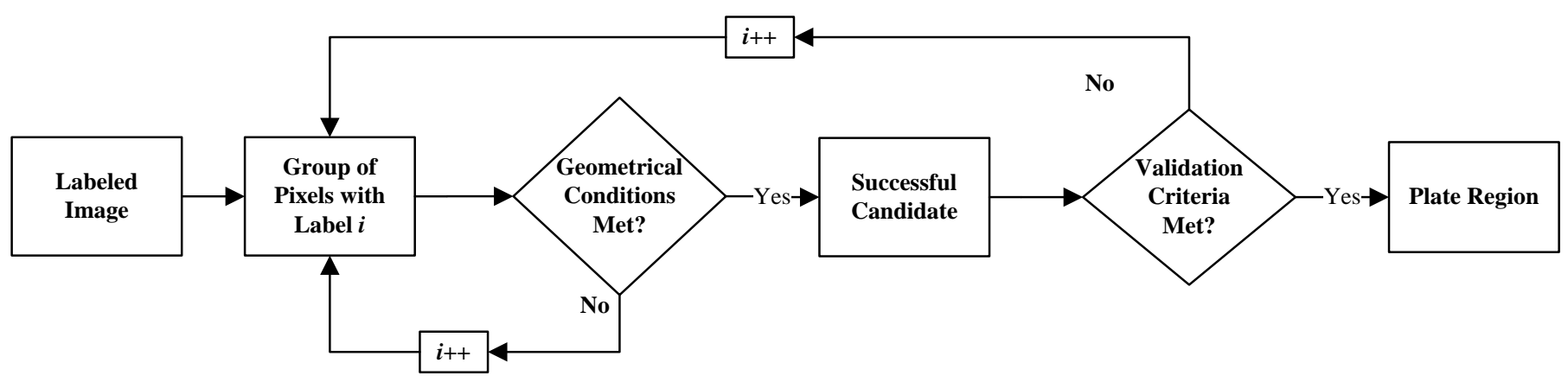

Figure 8. Chart of selection and validation processes.

Two geometrical conditions are used to select potential candidates. Let $\mathrm{P}$ denote the extracted plate region with the size $\mathrm{W} \times \mathrm{L}$, the first criterion is the ratio $\mathrm{r}$ between the width and length of $\mathrm{P}$ (i.e. $\mathrm{r}=\mathrm{L} / \mathrm{W}$ ). The second criterion is the ranges of $\mathrm{W}$ and $\mathrm{L}$. For this application, the ranges of $\mathrm{W}$ and $\mathrm{L}$ are large enough to cover most of the possible sizes of the plate region. These criteria can significantly reduce the number of candidate regions. However, there are few candidates that do not represent the actual plate. These candidates need to be filtered out using some validation criteria.

As the position of each potential candidate is known from the previous selection process, the validation criterion is mainly based on counting the number of connected component from each candidate from the complemented binary image. Only the connected components that meet the geometrical conditions of the character on the plate (e.g. width/length ratio of the character) will be taken into consideration. In theory for UK license plates, this number should be between 3 and 7 . In practice, in a true plate region this number is between 2 to 15 connected components and for a fake area is less than 2 or greater than 15 . Therefore, this method can be used to filter out the non number plate regions. Once acquiring the plate region coordinate, the final number plate can be extracted from the original binary image. Fig. 9 shows the validated plate region.

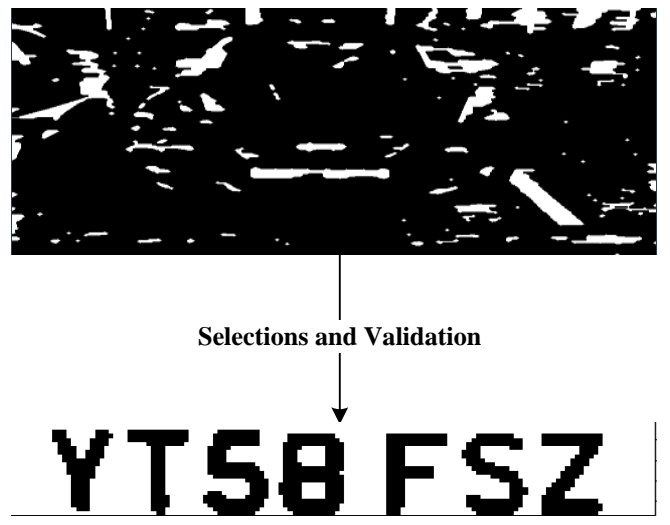

Figure 9. The plate region after validation.

\section{EXPERIMENTAL RESULTS AND DISCUSSION}

The proposed method was implemented and tested using Matlab. In order to analyse the performance of the proposed approach, a database of 1000 images was used for testing. These images were taken under different conditions, thus the database consists of blur, dark, complex scenes and inclined images which increases the complexity of testing. In addition, the images have low resolution (i.e. $768 \times 288$ ).

Fig. 10 shows some samples from the database that belongs to different categories: (a) is a blur image, (b) is an inclined image, (c) is an image with a complex background and (d) is a dark image.

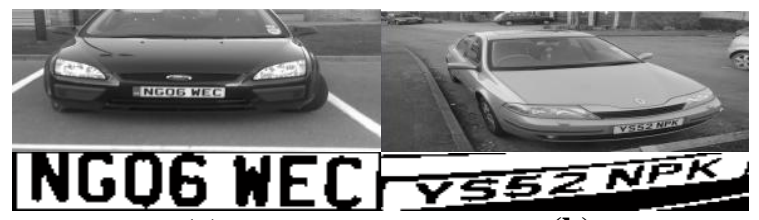

(a)

(b)

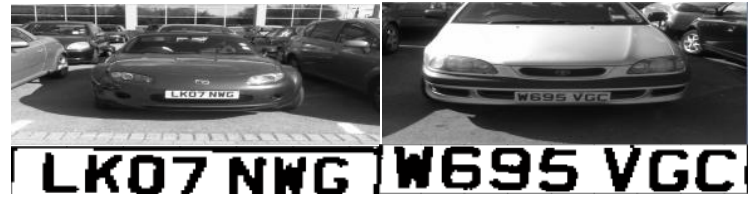

(c)

(d)

Figure 10. The original images and detected plates

The proposed method involves the use of only three morphologic operations which reduces the computational complexity compare to other existing methods such as the use of Gabor filter [11], global and local features [13] and Hough Transform [14]. In addition, the proposed method shows outstanding results in terms of the detection rate where most of licence plates in the images were detected successfully. Table 1 shows the performance comparison of the proposed method with other existing methods. 
TABLE I. DETECTION RATE COMPARISON

\begin{tabular}{|l|c|c|c|}
\cline { 2 - 4 } \multicolumn{1}{c|}{} & Database size & $\begin{array}{c}\text { Successful } \\
\text { Detections }\end{array}$ & $\begin{array}{c}\text { Detection } \\
\text { Rate }\end{array}$ \\
\hline Proposed method & 1000 & 987 & $98.7 \%$ \\
\hline Gabor filter in [11] & 300 & 294 & $98 \%$ \\
\hline $\begin{array}{l}\text { Global and local } \\
\text { features [13] }\end{array}$ & 169 & 158 & $93.5 \%$ \\
\hline $\begin{array}{l}\text { Morphology-based } \\
\text { method [4] }\end{array}$ & 130 & 128 & $98 \%$. \\
\hline
\end{tabular}

According to the analysis of the experimental results, there are typically four types of plate regions:
1. Complete plate region;
2. Incomplete plate region;
3. Declined plate region; and
4. Damaged plate region.

For the last three types, a pre-processing step is required to adjust or recover the plate regions for the next stage in the ANPR system (i.e. character recognition). For an incomplete plate region a recovery algorithm needs to be used to recover the missing parts of the plate. For a declined plate region, the image needs to be rotated where the rotated angle is calculated based on vertical projection value. For a damaged plate region, morphologic operations can be used to eliminate damages.

\section{CONCLUSIONS}

The demand of high performance and reliable ANPR systems has grown in current Intelligent Transportation Systems (ITSs). Locating the license plate in an image is the most important stage in such systems. Owing to the importance of Number Plate Localisation (NPL) in an ANPR system, an improved method based on morphologic operations to efficiently detect license plates has been proposed in this paper. The method has a low computational complexity and results achieved have shown outstanding detection rate compare to existing methods.

\section{REFERENCES}

[1] C. N. E. Anagnostopoulos, I. E. Anagostopoulos,V. Loumos et al. "A license plate-recognition algorithm for intelligent transportation system applications," IEEE Trans. Intell. Transp. Syst.7, pp. 377-391, 2006.

[2] F. Lian, Y. Fan, Y. Zhang, "Study of technology in electronic toll collection[J]," Compute Engineering and Application, Vol. 43(5), pp. 204-207, 2007.

[3] M. Vargas, S. L. Toral, F. Barrero, F. Cortés, “A license plate extraction algorithm based on edge statistics and region growing," International Conference on Image Analysis and Processing (ICIAP), pp. 317-326, 2009.

[4] J. Hsieh, S. Yu, and Y. Chen, "Morphology-based license plate detection from complex scenes," Proceedings of the 16 th International Conference on Pattern Recognition (ICPR'02) Volume 3, pp. 1051-4651. 2002.

[5] B. T. Cheon et.al., "The extraction of a number plate from a moving car," Proc. of First Workshop on Character Recognition, pp. 133-136. 1993.

[6] H. J. Cho, H. S. Chong, "Locating car license plate using subregion features," Journal of the KISS. Vol. 21 No. 6, pp. 1149-1159. 1994.

[7] S. Chang, L. Chen, Y. Chung, S. Chen, "Automatic license plate recognition," IEEE Transactions on Intelligent Transportation Systems. Vol. 5, pp. 42-53. 2004.

[8] G. Cao, J. Chen, J. Jiang, "An adaptive approach to vehicle license plate localization," The 29th Annual Conference of the IEEE Industrial Electronics Society. Vol. 2, pp. 1786-1791. 2003.

[9] K. I. Kim, K. Jung,J. H. Kim. "Color texture-based object detection:an application to license plate localization," Lecture Notes on Computer Science. Vol. 2388, pp. 293309, 2002.

[10] K. Kim, K. Jung,S. Park, H. Kim, "Support vector machines for texture classification," IEEE transactions on pattern analysis and machine intelligence. Vol. 24, No. 11, 2002.

[11] F. Kahraman, B. Kurt, and M. Gökmen. "License plate character segmentation based on the gabor transform and vector quantization," International Symposium on Circuits and Systems (ISCAS), pp.381-388, 2003.

[12] The MathWorks, Inc. Image Processing Toolbox 6 User Guide. 2009.

[13] H. Zhang, W. Jia, X. He and Q.Wu. "Learning-Based License Plate Detection Using Global and Local Features," International Journal of Intelligent Information and Database Systems (IJIIDS), Vol.1 No.2, pp. 228-243, 2007.

[14] Ganesan, V. Kamat and Subramaniam. "An Efficient Implementation of the Hough Transform for Detecting Vehicle License," Proceedings of Real-Time Technology and Applications, pp. 58-59, 1995. 